\title{
Parameterization of Joint Frequency Distributions of Potential Temperature and Water Vapor Mixing Ratio in the Daytime Convective Boundary Layer
}

\author{
LARRY K. BERG \\ Pacific Northwest National Laboratory, Richland, Washington \\ Roland B. STUlL \\ Atmospheric Science Programme, Department of Earth and Ocean Sciences, The University of British Columbia, Vancouver, \\ British Columbia, Canada
}

(Manuscript received 16 September 2002, in final form 3 November 2003)

\section{ABSTRACT}

\begin{abstract}
Joint frequency distributions (JFDs) of potential temperature $(\theta)$ versus water vapor mixing ratio $(r)$ within the convective boundary layer were measured during a new field experiment named Boundary Layer Experiment 1996 (BLX96). These JFDs were found to be tilted, with the tilt a function of both height and boundary layer dynamics. These distributions are also skewed and more peaked than a joint Gaussian distribution.

Three different methods are used to generate joint probability density functions (JPDFs) that approximate observed JFDs. Two classical methods, one based on a Gaussian fit and another based on surface-layer processes, are reviewed. A new method is devised, which treats the observed JFD as a mixing diagram. In the absence of advection, the only source regions for air in the mixing diagram are the surface and the entrainment zone. Thus, the tilt of the JFD can be explained by various mixtures from these two source regions. Methods that can be used to parameterize the mixing JPDF are presented. The primary advantage of this method is that the tilt is determined explicitly from properties of the surface, mixed layer, and entrainment zone.

Similarity methods are used to parameterize all variables needed by the Gaussian model. The Bowen ratio and the total energy input are used to parameterize the tilt of the surface energy budget JPDF, while similarity methods are used to define the spread of the JPDF along the two axes. Relationships between the surface and mixed layer, and the mixed layer and free atmosphere are used to tilt the mixing diagram JPDF, while similarity methods are used to estimate the spread of the JPDF. The parameterizations are developed using a "calibration" subset of data acquired during BLX96. A "verification" subset of data, also acquired during BLX96, is used to show that the parameterized mixing diagram method is superior to the other two methods, because it has either a smaller error or is less sensitive to the value of the correlation between $\theta$ and $r$.
\end{abstract}

\section{Introduction}

Joint frequency distributions (JFDs) provide an alternative method to investigate physical processes in the convective boundary layer. In addition, discrete JFDs provide data reduction of long time series by combining many observations into finite-sized bins. Holland (1973) used JFDs to investigate wave shapes. Mahrt and Paumier (1982) used JFDs of specific humidity and potential temperature $(\theta)$ to look at the effects of entrainment and mixing in a stratocumulus-topped boundary layer. Grossman (1984) used JFDs of vertical velocity $(w)$ and water vapor density to perform conditional sampling of data measured in a maritime boundary layer. Using data from the Air Mass Transformation Experiment, Mahrt

Corresponding author address: Dr. Larry K. Berg, Pacific Northwest National Laboratory, P.O. Box 999 MSIN K9-30, Richland, WA 99352.

E-mail: Larry.Berg@pnl.gov and Paumier (1984) constructed JFDs of $w$ and $\theta$. Deardorff and Willis (1985) examined JFDs of $w$ and temperature generated from their laboratory tank model. They also used JFDs to perform conditional sampling, looking for coherent structures in the boundary layer. Other authors (Lewellen and Yoh 1993; Xu and Randall 1996; Wang and Stevens 2000) have used finescale models to generate JFDs for cloudy boundary layers. Larson et al. (2001) examined distributions of conserved variables in cloudy boundary layers. Berg (2002) developed a cumulus parameterization that used subcloud distributions of $\theta$ versus $r$ to predict cloud cover and cloud size distributions.

A number of authors have examined the general shape of JFDs in the boundary layer. Lenschow (1970) suggested that observations of $\theta$ in the boundary layer are normally distributed, while Manton (1975) suggested that distributions of temperature are not normally distributed. Later work (e.g., Lenschow and Stephens 1980; Deardorff and Willis 1985; Cuijpers and Holtslag 1998; 
Wang and Stevens 2000) supported the contention by Manton that distributions of variables in the boundary layer are not normally distributed.

While JFDs have been used as tools to examine the convective boundary layer, there have been few attempts to quantify the shape of JFDs. There are two strategies that can be used to account for the tilt of the JFDs. The cross correlation between the two variables can be used to account for the tilt, or a coordinate transform can be used to describe the tilt of the JFD and eliminate the cross correlation. Wyngaard and Moeng (1992, hereafter WM92) suggested a statistical approach, using the correlation between $w$ and any arbitrary scalar to describe the tilt of the JFDs of $w$ and that scalar. They compared their theoretical joint probability density functions (JPDFs) to JFDs generated by a large eddy simulation (LES). Schrieber et al. (1996, hereafter SSZ) examined distributions of virtual potential temperature $\left(\theta_{v}\right)$ and moisture [their moisture variable was the height of the lifting condensation level $\left.\left(z_{\mathrm{LCL}}\right)\right]$. They took a different tack than WM92, developing a physically based method to perform a coordinate transformation to tilt the JPDFs and to eliminate the cross correlation observed in the surface layer.

A new conceptual model that can describe the tilt of JFDs of $\theta$ versus water vapor mixing ratio $(r)$ is proposed in this paper. Like the work of SSZ, this method is based on physical processes in the boundary layer. This approach views mixed-layer turbulence as a mixing process, and treats the JFD of $\theta$ versus $r$ as a mixing diagram (Paluch 1979; Hanson 1984; Betts 1984, 1985; Betts and Albrecht 1987). When advection is small, the mixed-layer distribution is a mixture of air from two different source regions, the surface layer and the free atmosphere. Thus, the tilt of the JFD can be determined if the thermodynamic properties of the surface, mixed layer, and entrainment zone are known. In this work, the properties of the mixed layer, the surface, and the free atmosphere are used to define a coordinate transformation to describe the tilt of Gaussian JPDFs. Data from Boundary Layer Experiment 1996 (BLX96) will be used to demonstrate that the new methods can successfully describe the size and shape of the JFDs in the convective mixed layer.

First, some definitions associated with the JFDs and JPDFs will be presented. Some relevant aspects of BLX96 will be reported in section 3. Observed JFDs measured during BLX96 are discussed next. The methods proposed by WM92 and SSZ and the new method are described in section 5. The methods used to determine the parameters derived from similarity theory are presented in section 6. Finally, in section 7, each of the three methods will be compared to a subset of BLX96 case study days.

\section{Definitions}

Joint frequency distributions count the occurrences of observations, $\hat{\theta}$ and $\hat{r}$, that lie within an interval $\Delta \theta$ and $\Delta r$ :

$$
\begin{aligned}
& F(\theta, r, \Delta \theta, \Delta r) \\
& \quad=\operatorname{count}(\theta<\hat{\theta}<\theta+\Delta \theta, r<\hat{r}<r+\Delta r) .
\end{aligned}
$$

Since every observation, $\hat{\theta}$ and $\hat{r}$, is somewhere within the whole $\theta-r$ "space," if there are $N$ observations, then

$$
\frac{1}{N} \sum_{\text {all observations }} F(\theta, r, \Delta \theta, \Delta r) \Delta \theta \Delta r \equiv 1 .
$$

Sometimes $1 / N$ is included with $F$ to make it a relative JFD, and sometimes $F$ is written as a frequency density that must be integrated over $\hat{\theta}$ and $\hat{r}$ to get the total count. If each of the variables has a normal distribution, then their joint normal distribution (written here as a JPDF) can be expressed as

$$
f(\theta, r)=\frac{1}{2 \pi \sigma_{\theta} \sigma_{r}\left(1-\rho_{\theta, r}^{2}\right)^{-1 / 2}} \exp \left\{\frac{-1}{2\left(1-\rho_{\theta, r}^{2}\right)}\left[\left(\frac{\theta-\bar{\theta}}{\sigma_{\theta}}\right)^{2}-\frac{2 \rho_{\theta, r}(\theta-\bar{\theta})(r-\bar{r})}{\sigma_{\theta} \sigma_{r}}+\left(\frac{r-\bar{r}}{\sigma_{r}}\right)^{2}\right]\right\},
$$

where $\rho_{\theta, r}$ is the correlation between $\theta$ and $r, \sigma_{\theta}$ is the standard deviation of $\theta, \sigma_{r}$ is the standard deviation of $r$, and the overbar represents a mean quantity. As indicated by (3), the interplay of the three parameters: $\rho_{\theta, r}$, $\sigma_{\theta}$, and $\sigma_{r}$ define the tilt of, and spread along, the axes of the JPDF.

\section{Boundary Layer Experiment 1996}

BLX96 was conducted between 15 July and 13 August 1996 over regions within the U.S. Department of Energy's Atmospheric Radiation Measurement (ARM) Southern Great Plains (SGP) Clouds and Radiation Test- bed (CART; Stokes and Schwartz 1994). This region includes parts of north-central Oklahoma and south-central Kansas. Details of BLX96 were reported by Stull et al. (1997) and Berg et al. (1997) and can be found online (see http://weather.eos.ubc.ca). A total of 12 research flights were flown during BLX96 using the University of Wyoming King Air aircraft. Each flight started between 1000 and 1030 LST, and lasted 4 to $4.5 \mathrm{~h}$. Only 10 flights were used for this study: 15, 16, 22, 23, 25, 27, 28, and 31 July and 2 and 13 August. The flight on 21 July was excluded because the tube leading to the fast response humidity sensor was not connected properly during the flight, 4 August was excluded because 
wind speeds (about $8 \mathrm{~m} \mathrm{~s}^{-1}$ ) led to forced, rather than free convection (Obukhov length of $-257.8 \mathrm{~m}$ ). On 16 July, the second half of the pattern was excluded because the measured moisture flux was suspect during the last near-surface leg (Berg 2002).

Three different locations within ARM SGP CART were chosen for BLX96 flights. The three legs were chosen to be over regions of different land use and to be close to at least one ARM SGP CART surface station, and are named after a nearby hamlet or town. The Lamont track was over flat terrain devoted to both wheat farming and hay production. Prior to BLX96 the wheat had been cut, and the wheat fields were bare. The ARM SGP CART central facility was located at the east end of this track. The Meeker track had many rolling hills and much forest coverage. The forest was not dense, and most of the trees were less than $10 \mathrm{~m}$ high. Some of the land was devoted to agriculture, either crops or hay production. The Winfield leg was over a region with some rolling hills. Most of the leg was over pasture, but there was some sparse forest near the east end of the track.

A unique flight pattern was designed for BLX96 to simultaneously satisfy three different experiments: a radix layer experiment (Santoso and Stull 1998, Santoso and Stull 2001), a convective transport theory experiment (Stull 1994), and work related to the JFDs and boundary layer cloud cover (Berg 2002). In each case, the pattern was oriented approximately perpendicular to the mean boundary layer flow. For all of the BLX96 flights, this resulted in tracks aligned in an east-west direction. Soundings were flown from near the surface to above cloud top 3 times during each flight; near the start, middle, and end of the pattern. Level horizontal legs about $72 \mathrm{~km}$ long were flown at a range of heights within the boundary layer. The highest horizontal leg was flown approximately $1000 \mathrm{~m}$ above ground level (AGL) or just below cloud base, whichever was lower. Other horizontal legs were flown at approximately 750 , 500, and $250 \mathrm{~m} \mathrm{AGL.} \mathrm{During} \mathrm{each} \mathrm{flight,} \mathrm{three} \mathrm{ap-}$ proximately terrain following near-surface legs were flown 30 to $60 \mathrm{~m}$ above the surface. Fluxes measured from these legs were used to compute standard boundary layer scales. The heights of all legs correspond to heights ranging from about $0.02 z_{i}$ to $0.8 z_{i}$, where $z_{i}$ is average mixed-layer depth.

A discrete Fourier transform (DFT) was used to filter the data collected from horizontal legs. A DFT was used, rather than a fast Fourier transform (FFT), to eliminate the need for zero padding or truncation of the data from each flight leg. Wavelengths less than approximately 20 $\mathrm{m}$ were removed from the data, primarily because the instruments on the aircraft were not collocated. Wavelengths greater than $5 \mathrm{~km}$ were removed to eliminate mesoscale effects that might be present, but that would have been poorly sampled. Variances and turbulent fluxes were computed using data with different long wavelength cutoffs, and the contribution by scales greater

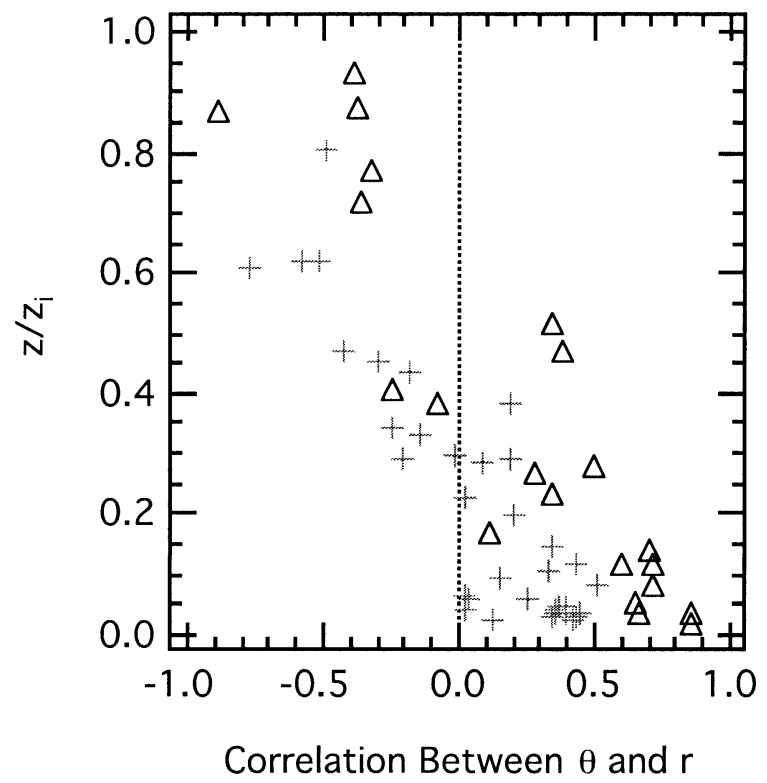

FIG. 1. Variation with dimensionless height of the correlation of $\theta$ and $r$ for BLX96 flight legs (crosses) and observations from Wyngaard et al. (1978; triangles) over the ocean.

than $5 \mathrm{~km}$ was generally small. DFTs or FFTs require that all data points be equally spaced and that there are no missing data. Replacing bad data is a challenge. In our case, the points identified as "bad" were not noticeably different than their neighbors; so they were not removed for the DFT filtering. However, these points were excluded for all subsequent calculations, for example calculations to determine the eddy-correlation flux or JFDs. To construct JFDs, each observation was placed into a $\theta$ versus $r$ bin $0.1 \mathrm{~K}$ by $0.1 \mathrm{~g} \mathrm{~kg}^{-1}$ wide.

\section{Observations}

While SSZ were the first to publish JFDs of $\theta_{v}$ versus $z_{\mathrm{LCL}}$, their data were limited to observations made approximately $100 \mathrm{~m}$ above the surface (roughly $0.05 z_{i}$ to $0.10 z_{i}$ ), and their flight legs averaged roughly $12 \mathrm{~km}$ in length. During BLX96, JFDs of $\theta$ versus $r$ were observed at heights ranging from $0.02 z_{i}$ to just above $0.8 z_{i}$, with most flight legs below $0.6 z_{i}$. Each BLX96 flight leg was approximately $70 \mathrm{~km}$ long. SSZ found a number of attributes were common to all JFDs; namely, they are bounded, they are sharply peaked, the spread is skewed along any axis of the distribution, they have a tilted axes, and a few JFDs have multiple peaks. The JFDs that were observed aloft during BLX96 share the key attributes described by SSZ for JFDs near the surface. However, there are some important differences. All the JFDs observed below $0.1 z_{i}$ have a positive correlation between $\theta$ and $r\left(\rho_{\theta, r}>0\right)$, in other words, there is a positive slope to the JFD (Fig. 1). There is also a large range in the observed $\rho_{\theta, r}$. Between $0.2 z_{i}$ and $0.4 z_{i}$ there is a transition zone where some, but not all the 

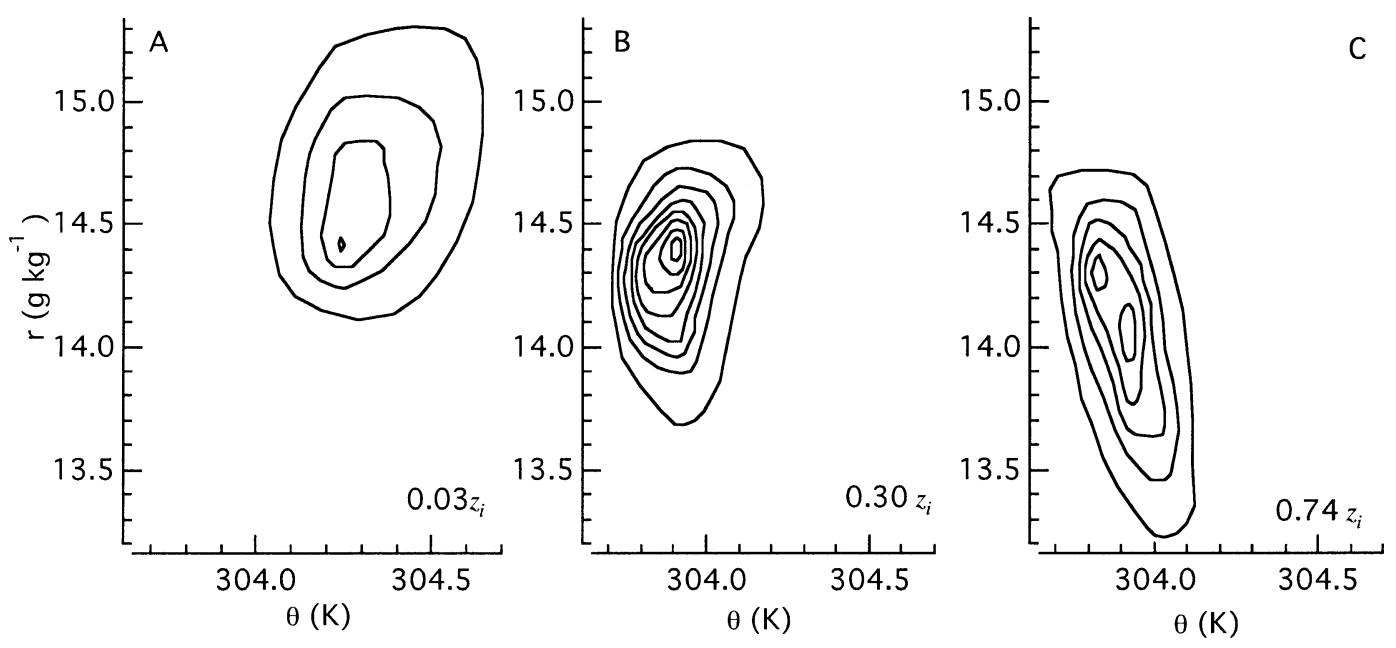

FIG. 2. Sequence of thee JFDs of $\theta$ vs $r$ at three different heights: (a) $0.03 z_{i}$, (b) $0.30 z_{i}$ and (c) $0.74 z_{i}$ measured on $16 \mathrm{Jul}$ 1996. The first contour indicates a normalized frequency of 0.005 , and the contour interval is 0.01 .

JFDs have a negative $\rho_{\theta, r}$, or in other words, a negative tilt. Above $0.4 z_{i}$, all of the observed JFDs have a negative $\rho_{\theta, r}$.

The change of $\rho_{\theta, r}$, or the change of tilt of the JFDs with height can be explained. Solar energy is absorbed at the earth's surface. Surface-layer processes cause air near the surface to warm and become moister. As these warm and moist parcels rise above the surface they cause the observed positive $\rho_{\theta, r}$. Near $z_{i}$, entrainment brings relatively warm and dry parcels from the free atmosphere down into the mixed layer. These warm and dry parcels lead to the observed negative $\rho_{\theta, r}$. Any method used to describe JFDs through the depth of the boundary layer must account for this behavior.

These measured values of $\rho_{\theta, r}$ can be compared to those reported by Wyngaard et al. (1978; Fig. 1). It appears that $\rho_{\theta, r}$ measured by Wyngaard et al. (1978) are larger near the surface than those measured during BLX96. Their measurements were from over the ocean where vigorous convection was driven by cold air flowing over warm water. In this scenario $\rho_{\theta, r}$ would be quite large because the surface layer is very moist. Over the middle of a continent, one would expect some of the thermals to be relatively dry and a smaller value of $\rho_{\theta, r}$ is expected. The observed differences between the results of Wyngaard et al. (1978) and BLX96 highlights that $\rho_{\theta, r}$ is not universal, but changes from time to time and place to place.

A sequence of JFDs observed on 16 July 1996 highlight this change of tilt (Fig. 2). Each JFD was separated in time by about $15 \mathrm{~min}$ and each leg took about 15 min to fly. No corrections for nonstationarity of the boundary layer between flight legs have been applied. This example is typical of JFDs measured at various heights during BLX96, the positive slope for the leg near the surface is obvious, as is the negative slope near the mixed-layer top. In this case, the middle leg seems to still have a positive tilt, but there is some evidence of a tail reaching toward warmer and dryer conditions. All of the JFDs observed during BLX96 were skewed. The average skewness of $\theta$ was 0.75 , while the average skewness of $r$ was -0.17 . The skewness was also a function of height. Each distribution was also more peaked than a Gaussian distribution. Figure 2 suggests that the lower-altitude JFDs might also have a smaller kurtosis ${ }^{1}$ than the upper-level legs. However, this trend disappears when all BLX96 case study days are considered.

\section{a. Identification of coherent drafts}

Coherent drafts will be used for the new method for describing the JFDs. A number of other researchers have investigated the convective boundary layer by locating coherent structures (e.g., Manton 1975; Coulman 1978; Lenschow and Stephens 1980; Nicholls and LeMone 1980; Greenhut and Khalsa 1982, 1987; Grossman 1984; Khalsa and Greenhut 1985; Young 1988; Williams and Hacker 1992; Schumann and Moeng 1991; Siebesma and Cuijpers 1995; Wang and Stevens 2000). Three different criteria are needed to identify the drafts: an indicator variable to define the thermal, some threshold value of the indicator variable, and some length scale. Unfortunately, all of these criteria are arbitrary. Either $\theta_{v}, T, r$, or $w$ have been used by various investigators as the indicator variable (Table 1). Crum and Stull (1987) tried to determine which indicator variable works the best. Based on observations of thermals made using a research aircraft and coincident lidar observations over land and near the mixed-layer top, they suggested that $r$ is the best choice to identify thermals. In

\footnotetext{
${ }^{1}$ Kurtosis has been defined relative to a Gaussian distribution (e.g., Press et al. 1992), so that a Guassian distribution would have a kurtosis of 0 .
} 
TABLE 1. List of indicator variables used in different field studies, the physical reasoning behing the choice of the variable, some potential shortfalls associated with that variable, and the researchers that have used that variable.

\begin{tabular}{|c|c|c|c|}
\hline Indicator variable & Physical reasoning & Potential shortfalls & Researchers \\
\hline$w$ & $\begin{array}{l}\text { Direct measure of thermal } \\
\text { velocity }\end{array}$ & Noisy signal & $\begin{array}{l}\text { Nicholls and LeMone (1980); Greenhut } \\
\text { and Khalsa (1982, 1987); Grossman } \\
\text { (1984); Khalsa and Greenhut (1985); } \\
\text { Young (1988); Siebesma and Cuijpers } \\
\text { (1995); Wang and Stevens (2000) }\end{array}$ \\
\hline$\theta_{v}$ & $\begin{array}{l}\text { Buoyancy is the dominant } \\
\text { force driving the thermal }\end{array}$ & $\begin{array}{l}\text { Other forces and inertia can be important, } \\
\text { especially high in the mixed layer where } \\
\text { the thermal is often less buoyant than its } \\
\text { surroundings }\end{array}$ & Williams and Hacker (1992) \\
\hline$r$ & $\begin{array}{l}\text { Thermals tend to be more } \\
\text { moist than the } \\
\text { environment }\end{array}$ & $\begin{array}{l}\text { May not always be true over land; } r \text { is } \\
\text { only weakly related to buoyancy }\end{array}$ & $\begin{array}{l}\text { Lenshow and Stephens (1980); Nicholls } \\
\text { and LeMone (1980); Crum and Stull } \\
\text { (1987); Siebesma and Cuijpers* } \\
\text { (1995); Wang and Stevens* (2000) }\end{array}$ \\
\hline$T$ & $\begin{array}{l}T \text { contributes substantially } \\
\text { to thermal buoyancy }\end{array}$ & $\begin{array}{l}r \text { also contributes to thermal buoyancy, } \\
\text { other forces can be important (see } \\
\text { discussion for } \theta_{v} \text { ) }\end{array}$ & Manton (1977); Coulman (1978) \\
\hline
\end{tabular}

* Siebesma and Cuijpers (1995) and Wang and Stevens (2000) used the liquid water content to identify updrafts, rather than mixing ratio.

contrast, Schumann and Moeng (1991) suggested that using $w$ to locate the thermals results in the most consistent results. Siebesma and Cuijpers (1995) used both $r$ and $w$ to define thermals in the cloudy boundary layer. In this study, $w$ will be used as an indicator variable because there was less scatter in the thermal statistics when using $w$ rather than $r$.

One common way to define the threshold is $\psi_{ \pm \text {thresh }}$ $=\sqrt{m_{\text {thresh }} \sigma_{ \pm \psi}^{2}}$, where $m_{\text {thresh }}$ is called the threshold multiplier (dimensionless) and $\psi$ is the indicator variable. The value of $\sigma_{ \pm \psi}^{2}$ is the variance of $\psi$ calculated using only the observations of $\psi$ greater than the mean value of $\psi$ (i.e., $\sigma_{+\psi}^{2}$ ), and the value of $\sigma_{-\psi}^{2}$ is the variance of $\psi$ calculated using only the observations of $\psi$ less than the mean value of $\psi$ (i.e., $\sigma_{-\psi}^{2}$ ). Following the lead of Greenhut and Khalsa (1982), the threshold value in this study was defined as $w_{ \pm \text {thresh }}=\sqrt{\sigma_{ \pm w}^{2} / 2}$. One advantage of this choice of $w_{ \pm \text {thresh }}$ is that the upward and downward mass flux are approximately the same (Greenhut and Khalsa 1982).

To be called a coherent draft, a structure must be horizontally wider than a specified length scale. In addition, if a distance less than the length scale separates two drafts, they are assumed to be one structure. Coulman (1978) used a length scale of zero, his only criterion was the $T$ threshold. Some authors (Greenhut and Khalsa 1982, 1987; Khalsa and Greenhut 1985) used a constant length scale. Young (1988) used a length scale of $0.1 z_{i}$. He chose this value because, in general, the smaller turbulence scales are within the inertial cascade. In our work, we will use a slightly smaller length scale of $0.05 z_{i}$. A number of BLX96 spectra, particularly near the surface, have a peak very close to $0.1 z_{i}$, and drop off at shorter scales (H. Modzelewski 2002, personal communication). Therefore, choosing a cutoff of $0.1 z_{i}$ might inappropriately remove some of the coherent drafts.

\section{b. Mixing diagrams}

JFDs of $\theta$ versus $r$ can be viewed as a mixing diagram because both $\theta$ and $r$ are conserved in the dry boundary layer. Mixing diagrams are useful because mixtures of air from two source regions lie on a straight line connecting the conservative properties of the two regions (Paluch 1979; Hanson 1984; Betts 1982, 1984, 1985; Betts and Albrecht 1987). As an example, mixing diagrams of data collected from the slant ascent/descent soundings flown during BLX96 can be examined. Figure 3 shows three sequential soundings measured on 27 July. A similar plot was presented by Betts (1992). This plot is typical of mixing diagrams generated from the BLX96 soundings.

Three different families of points are highlighted: mixed-layer points, entrainment-zone points and freeatmosphere points. On this particular day, $\theta$ and $r$ of the free atmosphere are found in the lower-right quadrant of the diagram. For this free-atmosphere air, the different $\theta$ values correspond to quasi-stationary conditions at different heights above ground. The mixed layer warmed with time (as shown by the shift to the right of the mixed-layer points in the top-left quadrant), and dried throughout the day (the mixed-layer points shifted down). The entrainment zone was determined from the slant ascent/descent soundings by locating the heights at which there were large jumps in the value of $r$. These large changes in $r$ are caused by dry freeatmosphere air intruding down into the moist convective boundary layer. This type of identification is relatively easy with aircraft slant soundings, which pass through a large horizontal distance (approximately $70 \mathrm{~km}$ during BLX96) and thus intersect many thermal circulations. It would be more difficult with balloon soundings that may drift along with a single thermal, and thus give effectively only a point sample. The points in the entrainment zone should lie on a mixing line between the 


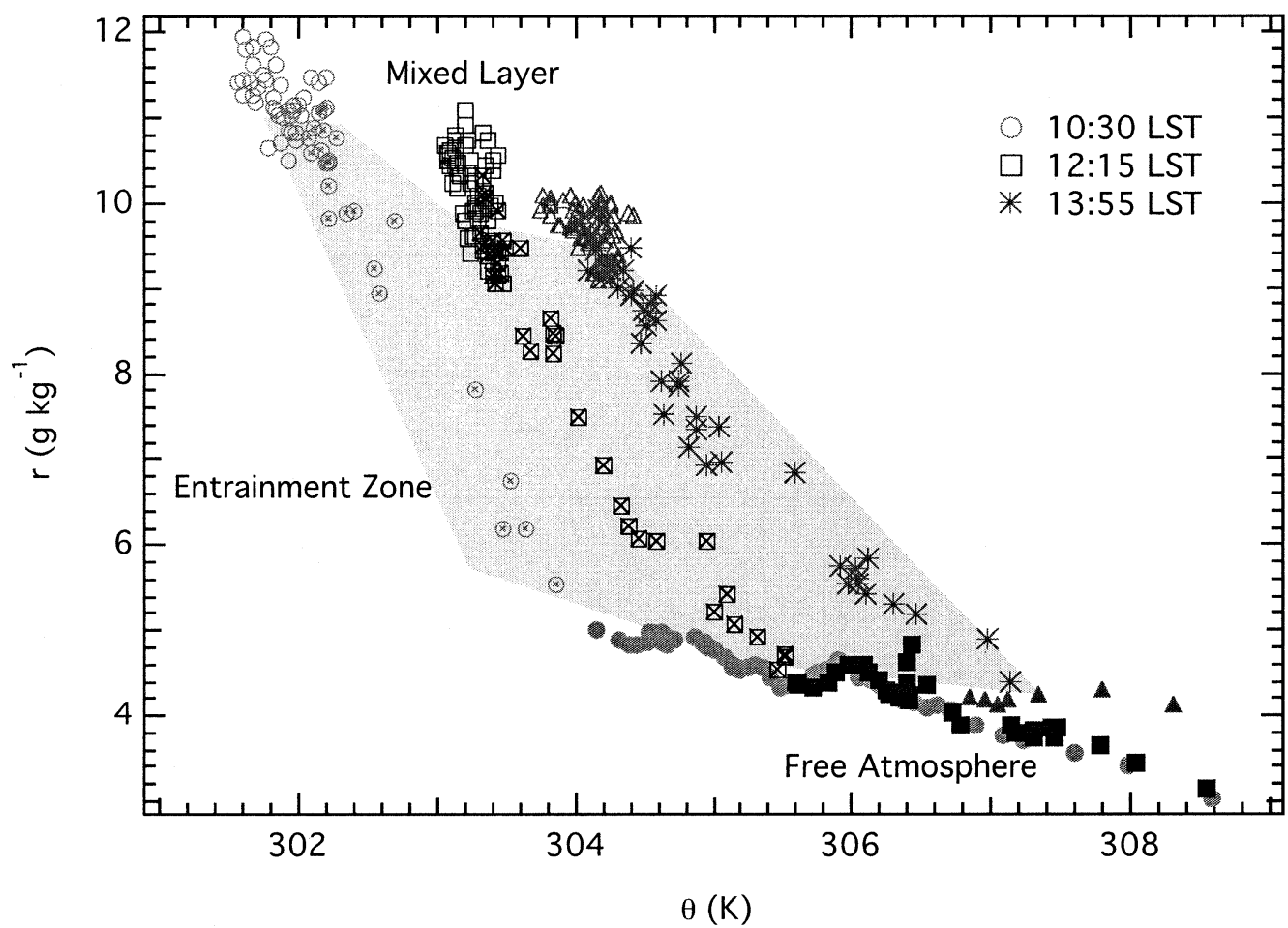

FIG. 3. Mixing diagrams for the three sequential soundings measured on 27 Jul. Each symbol represents a block average over a depth of $20 \mathrm{~m}$. The open symbols correspond to the mixed-layer values, circles with crosses, squares with crosses, and asterisks correspond to the entrainment-zone values, and the filled symbols correspond to the free atmosphere. Shading highlights observations that are part of the entrainment zone.

free-atmosphere values just above $z_{i}$, and the mixedlayer mean. That was the case for nearly all of the BLX96 soundings.

Surface-layer points are not highlighted in Fig. 3. For flight safety reasons, the soundings flown during BLX96 did not go low enough to capture much of the superadiabatic surface layer, so surface-layer properties were inferred from the updraft properties observed during any given leg in the following way. We assume that the mean properties of the observed updrafts lie on the mixing line that connects the mixed-layer point and the surface point on the mixing diagram. Therefore, these updraft properties can serve as a proxy for the surface-layer properties.

The mean thermodynamic properties of the updrafts, downdrafts, and environment can also be examined using a mixing diagram. An example, measured on 25 July, is plotted in Fig. 4. In this example, the mean updraft, downdraft, and environment air at any one time lie on a straight line or mixing line in $\theta$ versus $r$ space. This occurred for nearly all of the flight legs during BLX96. The only exceptions occurred on a few legs near the top of the mixed layer. If the mean thermodynamic properties of the updrafts, downdrafts and environment lie on a single mixing line, then $\left(\theta_{u}-\bar{\theta}\right) /$ $\left(r_{u}-\bar{r}\right) \approx\left(\theta_{u}-\theta_{d}\right) /\left(r_{u}-r_{d}\right)$, where $u$ indicates an updraft mean, $d$ indicates a downdraft mean, the overbar indicates a mixed-layer average. This information can be used in a new parameterization for JPDFs, as described in the next section.

\section{JPDF parameterizations}

\section{a. Classical methods}

Two methods have been suggested in the literature to parameterize JFDs in the boundary layer. WM92 fit joint Gaussian JPDFs to JFDs of an arbitrary scalar and $w$ generated by a LES model. They used three parameters to describe their JPDFs: the measured correlation between the scalar and $w$, the standard deviation of the scalar, and the standard deviation of $w$. Their method is statistical and did not offer a physical explanation for the tilt of the JFDs. While they constructed JPDFs of a scalar versus $w$, their mathematical techniques are also valid for JPDFs of $\theta$ versus $r$. Throughout the rest of this paper, this method will be called the statistical method. Three variables are needed to generate JPDFs using this method: $\sigma_{\theta}, \sigma_{r}$, and $\rho_{\theta, r}$.

SSZ introduced a physically based method to fit joint Gaussian JPDFs to JFDs of $\theta$ versus $r$ measured in the surface layer. They attempted to fit JPDFs by introducing two new, rotated axes to remove the correlation between $\theta$ and $r$. They related the slope of one axis to the Bowen ratio $(B)$, and the slope of the other axis to a variable they called the solar forcing $\left(\theta_{F}\right)$. While these 

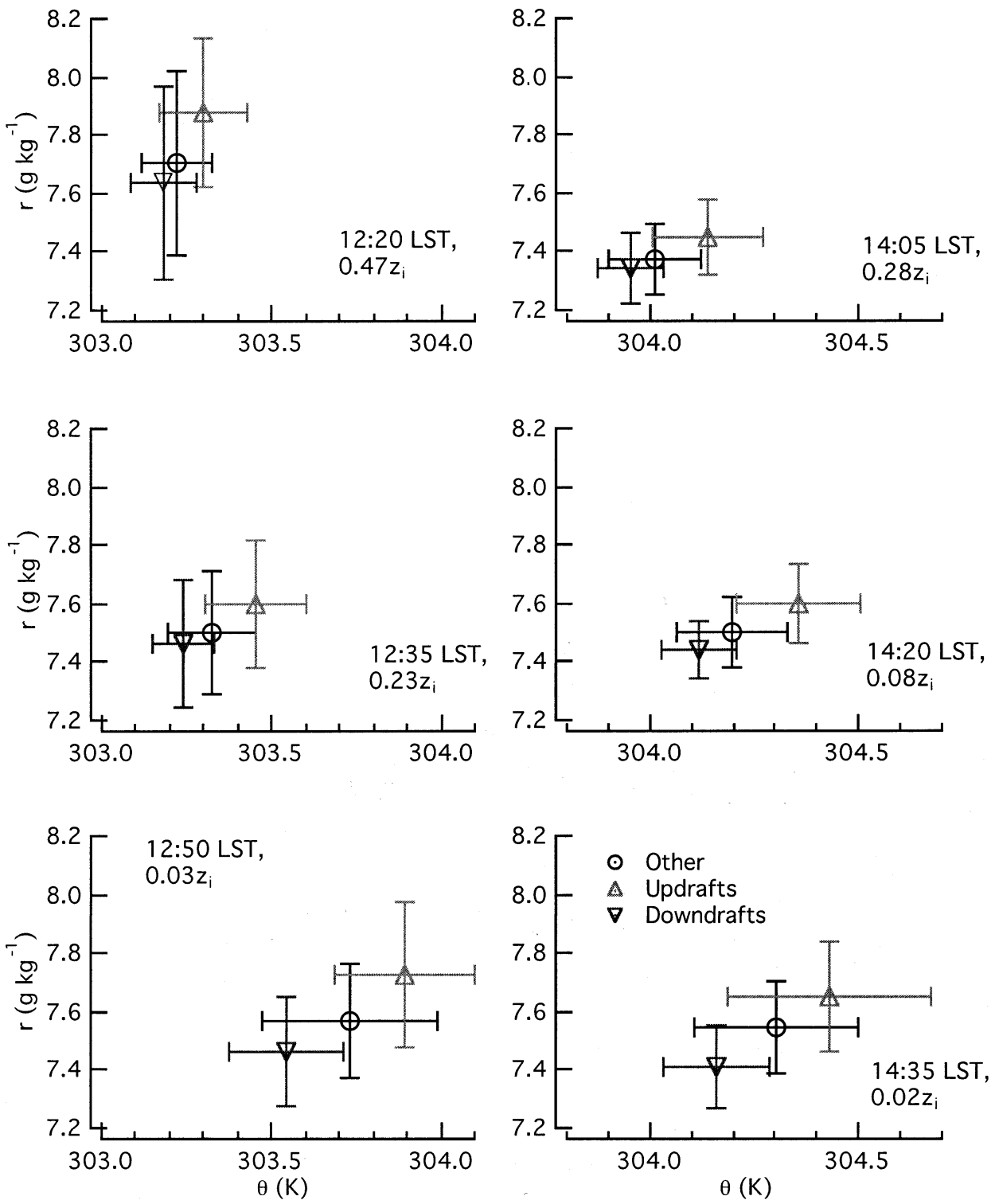

FIG. 4. Plot showing updraft (upward-pointing triangle), downdraft (downward-pointing triangle), and environment (circle) values of $\theta$ vs $r$ for six horizontal flight legs on 25 Jul. The error bars represent the standard deviations. Note the different $\theta$ scales between used for the left-hand and right-hand sides.

new axes are not geometrically orthogonal in $\theta$ versus $r$ space, changes along the $B$ axis are independent of changes along $\theta_{F}$ axes. This method will be called the $B-\theta_{F}$ method. Four variables are needed to generate JPDFs using this method, the slopes of the $B$ and $\theta_{F}$ axes, the standard deviation along the $B$ axis $\left(\sigma_{B}\right)$, and the standard deviation along the $\theta_{F}$ axis $\left(\sigma_{\theta_{F}}\right)$. In the appendix, SSZ's equations are converted from their $\theta_{v}$ versus $z_{\mathrm{LCL}}$ framework to the $\theta$ versus $r$ framework as used here.

\section{b. Mixing diagram method}

In the absence of advection, JFDs computed from observations made in the convective boundary layer are composed of a large number of mixtures of air originating at the surface, air that has been entrained from the free atmosphere, and air that has resided in the boundary layer for some time. Thus, the JFD should stretch along a mixing line toward the thermodynamic properties of both the surface and the entrainment zone. If advection is also considered, then the mixed-layer mixture is composed of air with three different origins (Fig. 5), which tend to "pull" the mixture off of a straight line between surface and entrainment-zone air. The primary advantage of this method is its basis on physical processes in the mixed layer. The primary disadvantage, compared to the statistical method, is the requirement of an additional parameter to compute the fit. 


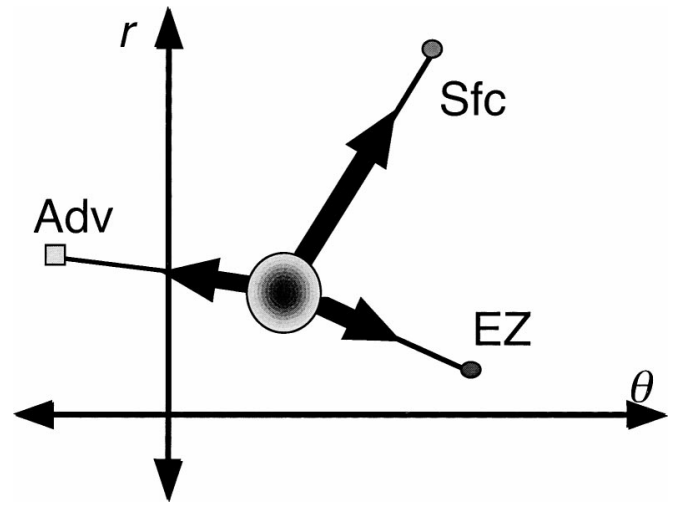

FIG. 5. Mixing diagram for $\theta$ vs $r$. The large circle represents the mixed-layer mean, the small circles represent the surface (Sfc) and entrainment-zone properties (EZ), and the square represents the advection (Adv). The large arrows indicate the "direction" that the different processes pull on the mixed-layer mean.

A coordinate transform is used to fit the mixing-diagram JPDFs to the JFDs and remove the correlation between $\theta$ and $r$. SSZ defined a transform to convert from $\theta$ and $r$ to a coordinate system defined using the slopes of their $B$ and $\theta_{F}$ axes. The transform is used to create a JPDF with two different axes that are tilted relative to the $\theta$ and $r$ axes. SSZ's definitions can be used to convert $\theta$ and $r$ data into a coordinate system, $c$ and $m$, based on slope of the surface axis $\left(S_{\text {sfc }}\right)$ and the slope of the entrainment-zone axis $\left(S_{\mathrm{ez}}\right)$ :

$$
c=S_{\mathrm{ez}}\left(\frac{\theta-S_{\mathrm{sfc}} r}{S_{\mathrm{ez}}-S_{\mathrm{sfc}}}\right) \text { and } m=\frac{\theta-S_{\mathrm{ez}} r}{S_{\mathrm{sfc}}-S_{\mathrm{ez}}},
$$

where $c$ has units of temperature and $m$ has units of mixing ratio.

The values of $c$ and $m$ can then be projected from the surface and entrainment zone axes onto the $\theta$ and $r$ axes. A joint Gaussian distribution, framed in $c$ versus $m$ coordinate system, takes the form

$$
f(m, c)=\frac{1}{2 \pi \sigma_{m} \sigma_{c}} \exp \left\{-\frac{1}{2}\left[\left(\frac{m}{\sigma_{m}}\right)^{2}+\left(\frac{c}{\sigma_{c}}\right)^{2}\right]\right\},
$$

where the correlation between $c$ and $m$ has been ignored. Before integrating (5) over $\theta$ and $r$, (5) should be multiplied by the Jacobian determinate, which is defined as

$$
|J(c, m)|=\left(\frac{\partial c}{\partial \theta} \frac{\partial m}{\partial r}-\frac{\partial m}{\partial \theta} \frac{\partial c}{\partial r}\right) .
$$

Using (4), it can be shown that $|J(c, m)|=S_{\mathrm{EZ}}\left(S_{\mathrm{EZ}}-\right.$ $\left.S_{\text {sfc }}\right)^{-1}$. Multiplication by $|J(c, m)|$ insures that the integral of the JPDF is equal to 1 . To use (5) with $c$ and $m$ defined by (4), one must first determine the axes slopes.

The surface axis slope $S_{\text {sfc }}$ is defined as

$$
S_{\mathrm{sfc}}=\left(\theta_{\mathrm{sfc}}-\bar{\theta}\right) /\left(r_{\mathrm{sfc}}-\bar{r}\right),
$$

where the overbar indicates a mean mixed-layer value and the subscript $s f c$ indicates a surface value. Equation (4) admits a special case (if $S_{\mathrm{sfc}}$ and $S_{\mathrm{ez}}$ are approximately equal, causing divide by zero) in which the mixing diagram method will fail because the two axes coincide as a single axis. In model forecasts, $S_{\text {sfc }}$ could be directly calculated using (7). An alternative expression for $S_{\mathrm{sfc}}$, which will be used here to parameterize the JPDFs, is based on the properties of the updrafts and the mixedlayer mean such that, $S_{\text {sfc }}=\left(\theta_{\text {up }}-\bar{\theta}\right) /\left(r_{\text {up }}-\bar{r}\right)$. It is tempting to define the slope of the entrainment zone axis in the same way as $S_{\text {sfc }}$. However, as shown in Fig. 3 , the entrainment zone cannot be adequately described using a single point. Rather $S_{\text {ez }}$ is defined using slope of a linear fit to observations of $\theta$ and $r$ made in the entrainment zone, which are highlighted in Fig. 3. In model forecasts, $S_{\text {ez }}$ could be inferred by the difference in $\theta$ and $r$ across the mixed-layer top.

An alternative expression for $S_{\text {sfc }}$, based on the Bowen ratio $(B)$, can be derived (SSZ). When $B$ is large, the sensible heat flux is large and the moisture flux is small; variations of $\theta$ tend to be large relative to variations of $r$, and $S_{\text {sfc }}$ is large. When $B$ is small, the heat flux is small relative to the latent heat flux, the variations of $\theta$ tend to be smaller than the variations of $r$, and $S_{\mathrm{sfc}}$ is small.

To investigate this approach further, it is convenient to define a new $B$ to be a function of height:

$$
B_{z}=\frac{C_{p} \overline{w^{\prime} \theta^{\prime}(z)}}{L_{v} \overline{w^{\prime} r^{\prime}(z)}}
$$

where the subscript $z$ indicates $B$ is a function of height, and where $C_{p}$ is the specific heat of air and $L_{v}$ is the latent heat of vaporization. Using the mass-flux approximation (e.g., Betts 1975, 1976; Manton 1975; Greenhut and Khalsa 1982, 1987; Nicholls and LeMone 1980; Randall et al. 1992; Siebesma and Cuijpers 1995) and ignoring the environmental flux, (8) can be written as

$$
B_{z} \approx \frac{C_{p}\left(\overline{\delta w_{u} \delta \theta_{u}} \alpha_{u}+\overline{\delta w_{d} \delta \theta_{d}} \alpha_{d}\right)}{L_{v}\left(\overline{\delta w_{u} \delta r_{u}} \alpha_{u}+\overline{\delta w_{d} \delta r_{d}} \alpha_{d}\right)},
$$

where $\overline{\delta w}$ is the average draft velocity, $\overline{\delta \theta}$ is the average draft perturbation value of $\theta, \overline{\delta r}$ is the average draft perturbation value of $r, \alpha$ is the fraction of legs covered by drafts, the subscript $u$ indicates updrafts, and the subscript $d$ indicates downdrafts. Equation (9) can be simplified because of mass continuity in the boundary layer, $\overline{\delta w_{u}} \alpha_{u}=\overline{\delta w_{d}} \alpha_{d}$, and rearranged to yield $S_{\mathrm{sfc}}$ :

$$
S_{\mathrm{sfc}}=\frac{\overline{\delta \theta_{u}}-\overline{\delta \theta_{d}}}{\overline{\delta r_{u}}+\overline{\delta r_{d}}}=\frac{L_{v} B_{z}}{C_{p}},
$$

which is similar to equations found by Betts (1984) and SSZ.

Figure 6 shows the observed slopes calculated from the observed updraft and downdraft properties observed during BLX96 and the slopes predicted by (10) using the value of $B_{z}$ calculated from the horizontal flight legs 


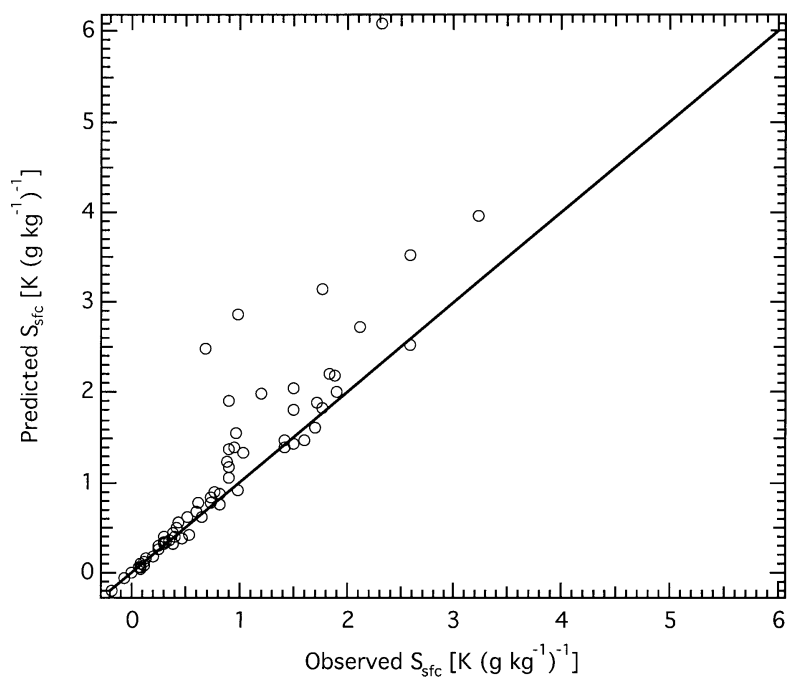

FIG. 6. Predicted surface axis slope using (10) (circles) vs observed surface axis slope for BLX96 case study days. The solid line is the 1:1 line.

over a range of heights (Fig. 6). A further simplification can be made to (10). In section $4 \mathrm{~b}$, it was shown that, $\left(\theta_{u}-\bar{\theta}\right) /\left(r_{u}-\bar{r}\right) \approx\left(\theta_{u}-\theta_{d}\right) /\left(r_{u}-r_{d}\right)$. This relationship can be combined with (10) so that the downdraft properties in (10) are replaced with the leg mean properties. There were some legs for which (10) do not do a good job. These cases were near the mixed-layer top where the mass-flux approximation did not do a good job representing the flux.

\section{Determination of parameters}

The variables that must be parameterized for each method are listed in Table 2. Those variables for which a physically based parameterization or a similarity parameterization will be developed are identified. The new parameterizations are derived for a subset of BLX96 case-study days chosen at random (Table 3 ). These "calibration subset" days happened to include legs from each location, although three of the five were the Winfield leg. Only one of the chosen days was clear. This random subset of days was chosen to allow the remaining case-study days to be used for verification of
TABLE 3. Calibration subset of BLX96 case-study days used to perform the statistical fits, and the associated location and cloudcover amount.

\begin{tabular}{cll}
\hline \hline Date & Location & Cu coverage \\
\hline $15 \mathrm{Jul}$ & Winfield & Scattered $\mathrm{Cu}$ \\
$22 \mathrm{Jul}$ & Winfield & None \\
$25 \mathrm{Jul}$ & Winfield & Scattered $\mathrm{Cu}$ \\
$27 \mathrm{Jul}$ & Lamont & Scattered $\mathrm{Cu}$ \\
$28 \mathrm{Jul}$ & Meeker & Scattered $\mathrm{Cu}$ \\
\hline
\end{tabular}

the results. All of our observations of $\sigma_{\theta} / \theta_{*}$ and $\sigma_{r} / r_{*}$, where $\theta_{*}$ is the Deardorff convective velocity scale and $r_{*}$ is a moisture scale, fall onto universal curves. Therefore, our results should not be sensitive to the choice of calibration and verification subsets. In addition, our research flights were conducted at three different locations each with different land-use characteristics, which makes our findings more robust.

\section{a. Statistical method}

Expressions are needed for $\sigma_{\theta}, \sigma_{r}$, and $\rho_{\theta, r}$ used by the statistical method. Many authors have shown that $\sigma_{\theta} / \theta_{*}$, and to a lesser extent $\sigma_{r} / r_{*}$ are well-behaved functions of height, when normalized by the appropriate scaling variable (e.g., Willis and Deardorff 1974; Lenschow and Stephens 1980; Khalsa and Greenhut 1987). Berg (2002) introduced a new moisture scaling variable $r_{* \text { new }}$, which is based on the moisture fluxes at both the top and bottom of the mixed layer:

$$
r_{*, \text { new }}(z)=\frac{\overline{w^{\prime} r_{\text {sc }}^{\prime}}\left(1-z / z_{i}\right)+\overline{w^{\prime} r_{z_{i}}^{\prime}}\left(z / z_{i}\right)}{w_{*}},
$$

where the subscript sfc indicates a surface value, and $z_{i}$ is the mixed-layer depth. It was shown in section 4 , that the correlation of $\theta$ and $r$ is also a function of height for BLX96. This behavior is consistent with the results reported by both Wyngaard et al (1978) and Wyngaard and LeMone (1980), although this relation may not be universal. These expressions can be used to parameterize the statistical method. Attempts to find an expression relating the correlation between $\theta$ and $r$ to Bowen ratio (B) were unsuccessful.

TABLE 2. List of methods used to model JFDs, the parameters needed, the basis for the parameterization (physical or similarity theory), and an associated equation number. The symbols have the following definitions: $S$ indicates the slope of an axis indicated by the subscript, $\sigma$ the spread along an axis indicated by the subscript, $\rho$ a correlation between two variables indicated by the subscripts, a subscript "sfc" indicates surface axis, a subscript "ez" indicates entrainment-zone axis, a subscript $B$ indicates Bowen ratio axis, and a subscript $\theta_{F}$ indicates solar-forcing axis.

\begin{tabular}{|c|c|c|c|}
\hline Method & Parameters needed & Physically based & Similarity based \\
\hline Statistical & $\bar{\theta}, \bar{r}, \sigma_{\theta}, \sigma_{r}$, and $\rho_{\theta, r}$ & & $\sigma_{\theta}(12), \sigma_{r}(13)$, and $\rho_{\theta, r}(14)$ \\
\hline$B-\theta_{F}$ & $\bar{\theta}, \bar{r}, \sigma_{B}, \sigma_{\theta_{F}}, S_{B}$, and $S_{\theta_{F}}$ & $S_{B}$ and $S_{\theta_{F}}$ & $\begin{array}{l}\sigma_{B}[(15 a) \text { or }(16 a)] \text { and } \sigma_{\theta_{F}}[(15 b) \\
\quad \text { or }(16 b)]\end{array}$ \\
\hline Mixing diagram & $\bar{\theta}, \bar{r}, \sigma_{\mathrm{sfc}}, \sigma_{\mathrm{ez}}, S_{\mathrm{sfc}}$, and $S_{\mathrm{ez}}$ & $\begin{array}{l}S_{\mathrm{sfc}}[(7) \text { or }(10)] \text { and } S_{\mathrm{ez}}(\mathrm{com}- \\
\text { puted from sounding) }\end{array}$ & $\begin{array}{l}\sigma_{\text {sfc }}[(17 a) \text { or }(18 a)] \text { and } \sigma_{\text {ez }}[(17 b) \\
\quad \text { or }(18 b)]\end{array}$ \\
\hline
\end{tabular}




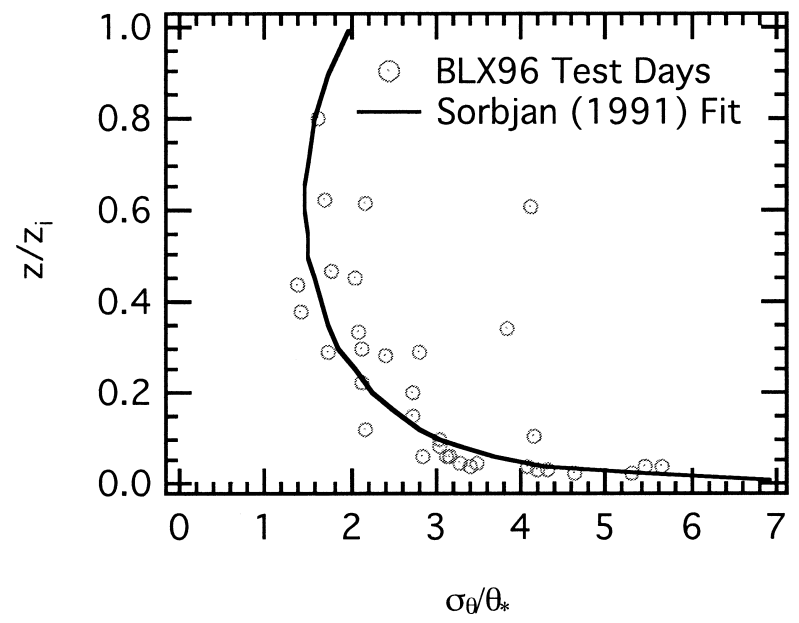

FIG. 7. Sorbjan's expression for $\sigma_{\theta} / \theta_{*}$ (line) and observed values of $\sigma_{\theta} / \theta_{*}$ (symbols) vs $z / z_{i}$ for the subset of BLX96 case-study days.

Sorbjan (1991) suggested an expression for $\sigma_{\theta} / \theta_{*}$ [his Eq. (6)]:

$\frac{\sigma_{\theta}}{\theta_{*}}=\left[c_{2 b} \frac{\left(1-z / z_{i}\right)^{4 / 3}}{\left(z / z_{i}\right)^{2 / 3}}+c_{2 t} R^{4 / 3} \frac{\left(z / z_{i}\right)^{4 / 3}}{\left(1-z / z_{i}+D\right)^{2 / 3}}\right]$,

where $R$ is the Ball ratio (Ball 1960) of entrainmentzone heat flux to surface heat flux, $D$ is the ratio of the entrainment zone depth to $z_{i}$, and $c_{2 b}$ and $c_{2 t}$ are empirical constants. Sorbjan suggests that $c_{2 b}=2.2$ and $c_{2 t}=6$. For the calibration subset of BLX96 days, the average value of $R$ was 0.46 and the value of $D$ was 0.4. Figure 7 shows the results from (12) and the BLX96 data using these average values. Sorbjan's expression does a good job representing BLX96 data, although it may underestimate $\sigma_{\theta} / \theta_{*}$ higher in the mixed layer. This relationship, which is not adjusted to fit BLX96 data, is used for all subsequent calculations of $\sigma_{\theta} / \theta_{*}$.

Sorbjan (1991) also derived an expression for $\sigma_{r} / r_{*}$. However, as was shown by Berg (2002), the traditional value of $r_{*}$ is not the best choice, so the new expression (11) was derived. Berg (2002) shows that the new scaling does a good job collapsing the BLX96 moisture data to a universal curve (Fig. 8). For the calibration subset of BLX96 data, the least squares best-fit expression for the curve $\sigma_{r} / r_{* \text {,new }}$ is

$$
\sigma_{r} / r_{*, \text { new }}=2.8+0.25\left(z / z_{i}\right)^{-0.81} .
$$

Expressions are also needed for $\rho_{\theta, r}$. The data from BLX96 show a linear variation of $\rho_{\theta, r}$ with height, so a straight line was fit to the observations (Fig. 1). The best-fit line for the BLX96 data was found to be

$$
\rho_{\theta, r}=0.39-1.4\left(z / z_{i}\right) .
$$

As pointed out in section 4, and shown in Fig. 3, it appears that this curve is not universal. Wyngaard et al. (1978) reported values of $\rho_{\theta, r}$ measured in the maritime boundary layer that were larger than those found during BLX96. Some implications of the variation of $\rho_{\theta, r}$ for different parameterizations will be discussed in section 7 .

\section{b. $B-\theta_{F}$ method}

Expressions are needed for tilt of the axes, and for the spread along the $B$ and $\theta_{F}$ axes $\left[\sigma_{B}\left(\mathrm{~g} \mathrm{~kg}^{-1}\right)\right.$ and $\sigma_{\theta_{F}}(K)$, respectively] used by the $B-\theta_{F}$ method. Data from the calibration subset of BLX96 flights was used to calculate $\sigma_{B}$ and $\sigma_{\theta_{F}}$. Figure 9 shows that, when normalized by $\theta_{*}$ or $r_{* \text {,new }}$, the observed values of $\sigma_{B}$ and $\sigma_{\theta_{F}}$ each collapse toward a single curve. Using a least squares technique, the best-fit power law was found to be

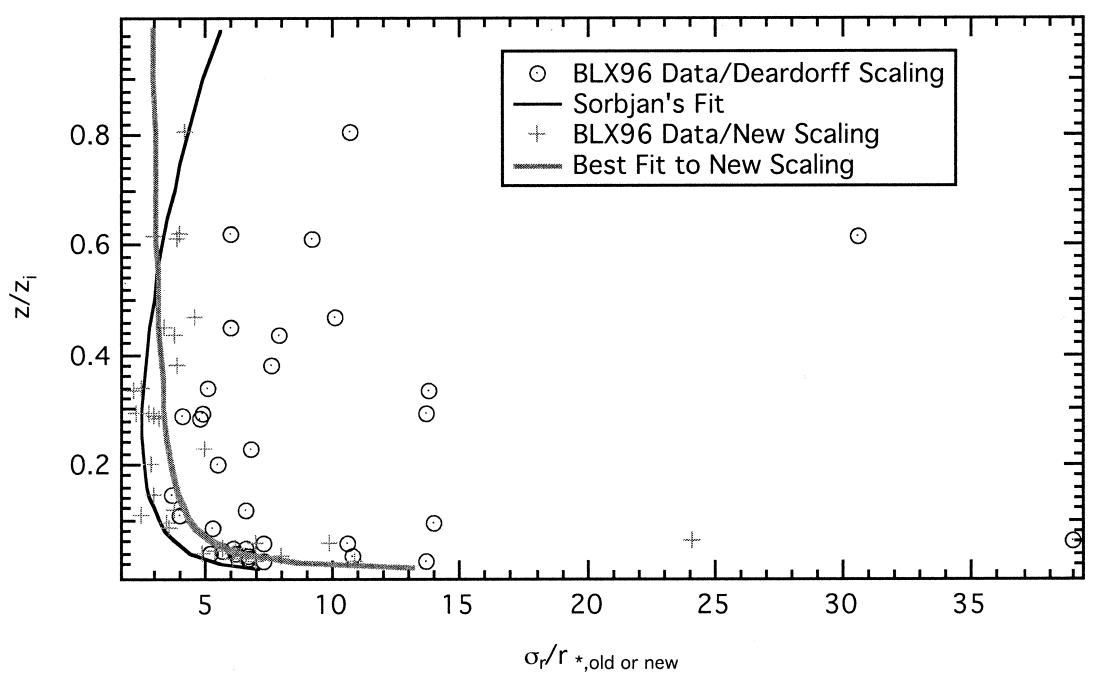

FIG. 8. Sorbjan's expression for $\sigma_{r} / r_{*}$ (thin line), BLX96 data scaled with standard Deardorff scaling, (circles), BLX96 data scaled with $r_{*, \text { new }}$ (crosses), and BLX96 best fit (13) (thick line) vs $z / z_{i}$. 

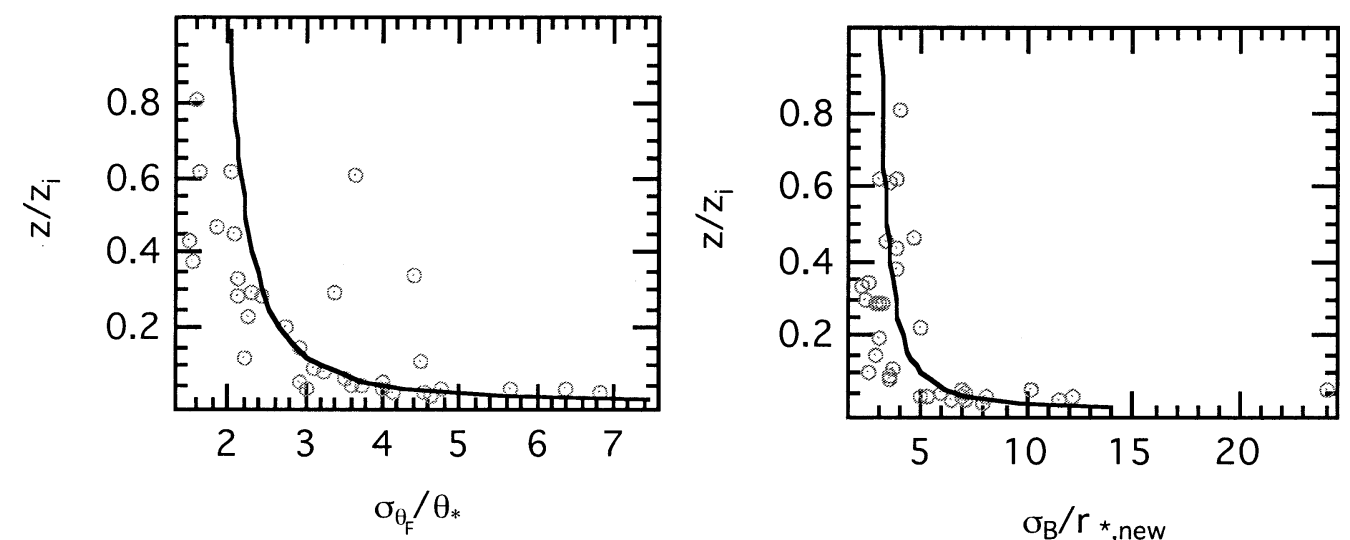

FIG. 9. Observed values of (left) $\sigma_{\theta_{r}} / \theta_{*}$ (circles), (right) $\sigma_{B} / r_{* \text {,new }}$ (circles), and best-fit curves [(15a) and (b)] (lines) for the BLX96 control days vs $z / z_{i}$.

$$
\begin{aligned}
\sigma_{B} / r_{*, \text { new }} & =2.53+0.57\left(z / z_{i}\right)^{-0.65}, \text { and } \\
\sigma_{\theta_{F}} / \theta_{*} & =1.56+0.47\left(z / z_{i}\right)^{-0.56} .
\end{aligned}
$$

Alternatively, equations for $\sigma_{B}$ and $\sigma_{\theta_{F}}$ were derived by SSZ that were based on $\sigma_{\theta}, \sigma_{r}$, and $\rho_{\theta, r}$, yielding

$$
\sigma_{B}^{2}=\left(\frac{1}{S_{B}-S_{\theta_{F}}}\right)^{2}\left(\sigma_{\theta}^{2}-2 S_{\theta_{F}} \rho_{\theta, r} \sigma_{\theta} \sigma_{r}+S_{\theta_{F}}^{2} \sigma_{r}^{2}\right), \quad \text { and }
$$

$\sigma_{\theta_{F}}^{2}=\left(\frac{S_{\theta_{F}}}{S_{B}-S_{\theta_{F}}}\right)^{2}\left(\sigma_{\theta}^{2}-2 S_{B} \rho_{\theta, r} \sigma_{\theta} \sigma_{r}+S_{B}^{2} \sigma_{r}^{2}\right)$,

where $S_{B}$ is the slope of the $B$ axis and $S_{\theta_{F}}$ is the slope of the $\theta_{F}$ axis. Equations (16a) and (16b) were used to generate the values of $\sigma_{B}$ and $\sigma_{\theta_{F}}$ used in this study, rather than (15a) and (15b), so that results from the $B-$ $\theta_{F}$ and the statistical method are directly comparable. In other words, differences in the fits are due to differences in theories, rather than differences in the predicted value of the standard deviations. Values of $\sigma_{\theta}, \sigma_{r}$ and $\rho_{\theta, r}$ needed by (16a) and (16b) were calculated from (12), (13), and (14).

\section{c. Mixing diagram method}

The mixing-diagram method requires parameterization of the following: the slope of the surface axis $\left(S_{\mathrm{sfc}}\right)$, the slope of the entrainment-zone axis $\left(S_{\mathrm{ez}}\right)$, the standard deviation along the surface axis $\left[\sigma_{\mathrm{sfc}},\left(\mathrm{g} \mathrm{kg}^{-1}\right)\right]$, and the standard deviation along the entrainment-zone axis $\left[\sigma_{\mathrm{ez}}\right.$, $(K)$ ]. Only $\sigma_{\text {sfc }}$ and $\sigma_{\text {ez }}$ need to be parameterized using similarity methods because $S_{\text {sfc }}$ and $S_{\text {ez }}$ are either known from theory or model output.

Using data from the BLX96 calibration subset days, least-square best-fit power-law functions can be fit to the curves of $\sigma_{\mathrm{ez}} / \theta_{*}$ and $\sigma_{\mathrm{sfc}} / r_{* \text {,new }}$ :

$$
\begin{aligned}
\sigma_{\mathrm{ez}} / \theta_{*} & =2.0+8.2 \times 10^{-4}\left(z / z_{i}\right)^{-1.8} \text { and } \\
\sigma_{\text {sfc }} / r_{*, \text { new }} & =2.3+1.1 \times 10^{-2}\left(z / z_{i}\right)^{-1.6} .
\end{aligned}
$$

Figure 10 shows (17a) and (17b) plotted as a function
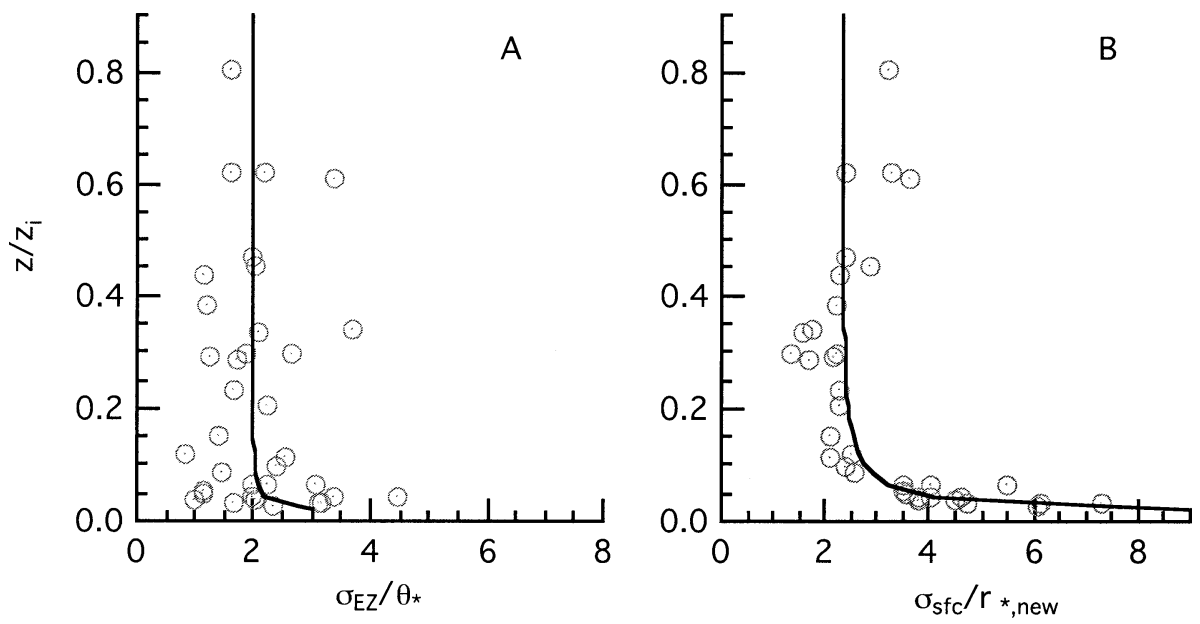

FIG. 10. Observed (a) $\sigma_{\mathrm{ez}} / \theta_{*}(17 \mathrm{a})$ and (b) $\sigma_{\text {sfc }} / r_{* \text {,new }}(17 \mathrm{~b})$ on the BLX96 case study days vs $z / z_{i}$. 
TABLE 4. Verification subset of BLX96 case-study days used to perform the statistical fits, and the associated location and cloudcover amount.

\begin{tabular}{cll}
\hline \hline Date & Location & Cu coverage \\
\hline $16 \mathrm{Jul}$ & Meeker & Scattered $\mathrm{Cu}$ \\
$23 \mathrm{Jul}$ & Winfield & Scattered $\mathrm{Cu}$ \\
$31 \mathrm{Jul}$ & Winfield & Scattered $\mathrm{Cu}$ \\
$2 \mathrm{Aug}$ & Meeker & None \\
$13 \mathrm{Aug}$ & Lamont & None \\
\hline
\end{tabular}

of $z / z_{i}$. The agreement seems to be good for the range of $z / z_{i}$ observed during BLX96. However, it is not clear that $\theta_{*}$ and $r_{* \text {,new }}$ are the relevant scales. While the value of $\sigma_{\mathrm{ez}}$ does have units of temperature, it represents a relationship that is dependant on both $\theta$ and $r$. Likewise, $\sigma_{\text {sfc }}$ is also a function of both $\theta$ and $r$. Efforts were made to define two new scaling variables using the projected variables $c$ and $m$. First, the observed heat and moisture fluxes were converted to fluxes of $c$ and $m$ using SSZ's (14a) and (14b). The fluxes of $c$ and $m$, along with $w_{*}$ were used to define $c_{*}$ and $m_{*}$ using definitions analogous to those used by standard Deardorff scaling. Unfortunately, this scaling did not improve the results.

As an alternative to (17a) and (17b), $\sigma_{\text {sfc }}$ and $\sigma_{\text {ez }}$ can be defined in terms of $\sigma_{\theta}, \sigma_{r}$, and $\rho_{\theta, r}$ using definitions derived by SSZ as

$$
\sigma_{\mathrm{ez}}^{2}=\left(\frac{S_{\mathrm{sfc}}}{S_{\mathrm{ez}}-S_{\mathrm{sfc}}}\right)^{2}\left(\sigma_{\theta}^{2}-2 S_{\mathrm{sfc}} \rho_{\theta, r} \sigma_{\theta} \sigma_{r}+S_{\mathrm{sfc}}^{2} \sigma_{r}^{2}\right), \text { and }
$$

$\sigma_{\mathrm{sfc}}^{2}=\left(\frac{1}{S_{\mathrm{ez}}-S_{\mathrm{sfc}}}\right)^{2}\left(\sigma_{\theta}^{2}-2 S_{\mathrm{ez}} \rho_{\theta, r} \sigma_{\theta} \sigma_{r}+S_{\mathrm{ez}}^{2} \sigma_{r}^{2}\right)$.

Thus, the value of $\sigma_{\mathrm{sfc}}, \sigma_{\mathrm{ez}}$, and $\rho_{\theta, r}$ can be found from a combination of (18) with (12), (13), and (14) to account for the influences of both $\theta$ and $r$ in each standard deviation. As described in the previous section, (18a) and (18b) give results that are more consistent with the methods used to fit the statistical method.

\section{Verification against independent data}

A "verification subset" was created from all the remaining BLX96 cases that were not previously chosen at random to help with calibration. The verification subset is listed in Table 4. JPDFs were created for the verification subset of BLX96 cases using theories presented in section 5 and the equations presented in section 6. Namely, values of $\sigma_{\theta}, \sigma_{r}$, and $\rho_{\theta, r}$ needed by the statistical method were determined from (12), (13), and (14), respectively. SSZ's theories were used to predict $S_{B}$ and $S_{\theta_{F}}$ for the $B-\theta_{F}$ method. Equations (16a) and (16b) were used to predict $\sigma_{B}$ and $\sigma_{\theta_{F}}$, respectively. For the mixing diagram method, the value of $S_{\text {sfc }}$ was predicted from (10), while $\sigma_{\mathrm{sfc}}$ and $\sigma_{\mathrm{ez}}$ were predicted from Eqs. (18a) and (18b). Relevant soundings were used to calculate $S_{\mathrm{ez}}$ by fitting a line to the entrainment zone observations, and assuming $S_{\mathrm{ez}}$ varied linearly in time between the soundings.

The similarity relationships derived in section 6 [Eqs. (15a), (15b), (17a), and (17b)] could have been used to find $\sigma_{B}, \sigma_{\theta_{F}}, \sigma_{\mathrm{ez}}$, and $\sigma_{\mathrm{sfc}}$ based on observations from BLX96. However, using (16a), (16b), (18a), and (18b), which are formulated in terms of $\sigma_{\theta}, \sigma_{r}$, and $\rho_{\theta, r}$, to predict the values of $\sigma_{B}, \sigma_{\theta_{F}}, \sigma_{\mathrm{ez}}$, and $\sigma_{\text {sfc }}$ has an advantage: results from each method are more consistent with each other. In other words, differences in the fits are due to differences in the theories, rather than differences in the predicted value of the standard deviations.

Figure 11 shows sample fits for a single leg flown on 27 July 1996. This leg was flown at a height of $0.62 z_{i}$. In this case, the tilt of the JFD is negative. The $B-\theta_{F}$ method is unable to capture this behavior, while both the statistical and mixing diagram methods can. For this case the difference between the statistical method and the mixing diagram method is very small. The peak frequency of all methods is less than the observed JFD, although in this example the differences are small. The observed peak frequency was 0.083 , while the predicted frequencies for the statistical, $B-\theta_{F}$, and mixing diagram method were $0.079,0.060$, and 0.079 , respectively. Similar results were found for all BLX96 JPDFs; namely, each method underestimated the peak frequency.

JPDFs were computed for all the BLX96 verification subset cases, and the error of each method was computed using the method of SSZ:

$$
\text { err }=\sum\left|\hat{f}_{\text {obs }, j}-\hat{f}_{\text {param }, j}\right|,
$$

where $\hat{f}_{\text {obsj }}$ is the normalized frequency observed for the $j$ th bin, and $\hat{f}_{\text {param. } j}$ is the normalized frequency from the $j$ th bin of the parameterization. The results from each method were complied and are shown in Fig. 12. It appears that both the statistical method and the mixing diagram method work better than the $B-\theta_{F}$ method. There is only one case where the error from the $B-\theta_{F}$ method is close to the one-to-one line. There is little difference in the error predicted by the statistical method and the mixing diagram method; most of the points are very close to the 1:1 line. As pointed out by SSZ, an error of 1.0 shows no skill. Both the statistical and mixing diagram method are better than no fit for most of the flight legs; only one flight leg had an error in the statistical or mixing diagram method that was greater than 1.0. Many of the $B-\theta_{F}$ JPDFs had errors larger than 1.0, Berg (2002) attributed these errors to shortcomings associated with the prediction of the slopes $S_{B}$ and $S_{\theta_{F}}$.

The good agreement between the statistical method and the mixing diagram method is encouraging. For BLX96 case-study days the statistical method should do a good job because each of the parameters $-\sigma_{\theta}, \sigma_{r}$, and $\rho_{\theta, r}$-are well behaved. However, the mixing diagram method has several advantages over the statistical method. First, the mixing diagram method is more robust 

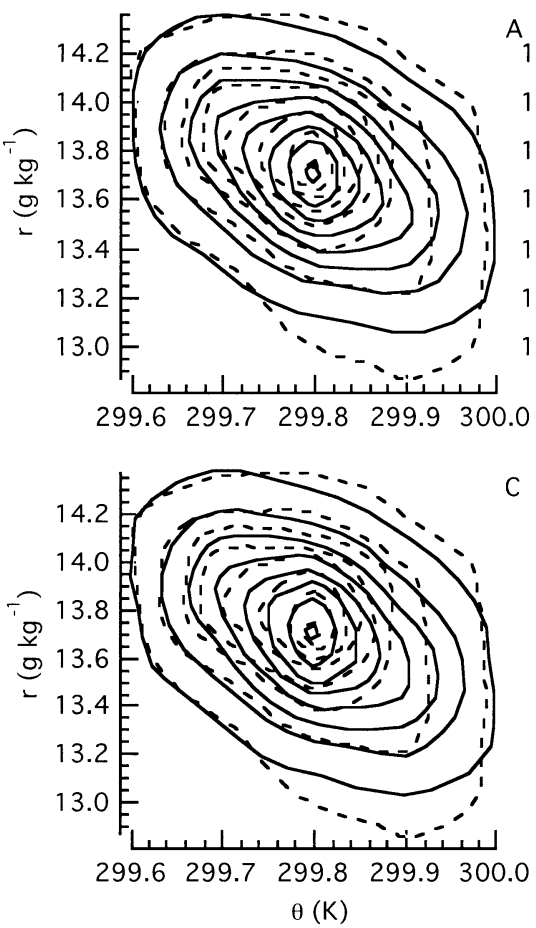

FIG. 11. Observed (broken lines) JFDs and parameterized (solid lines) JPDFs using (a) statistical, (b) $B-\theta_{F}$, and (c) mixing diagram methods for $27 \mathrm{Jul} 1996$, where $z / z_{i}=0.62$ at 1211 LST

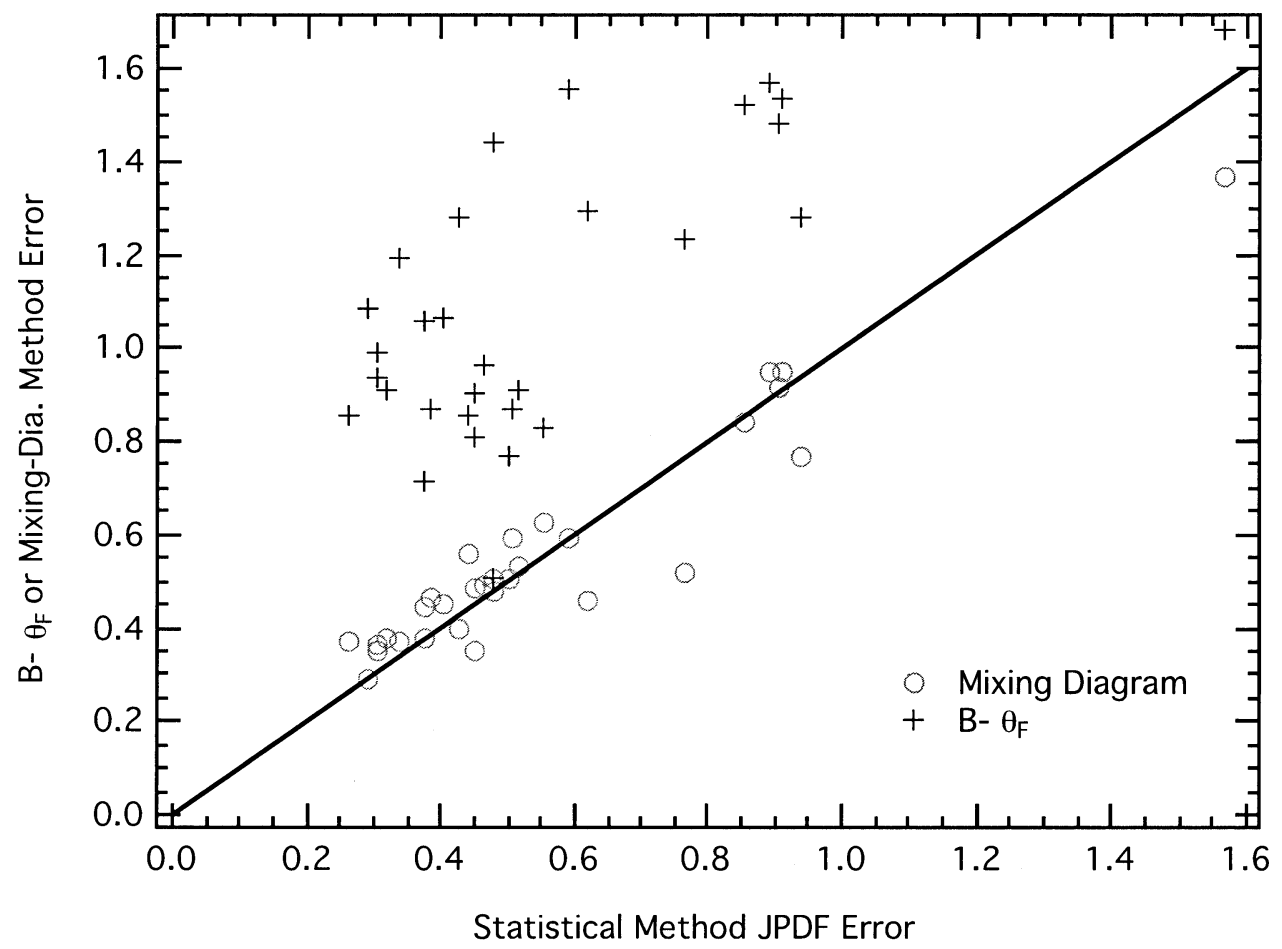

FIG. 12. Errors associated with the parameterized statistical method JPDF vs error associated with either the mixing diagram method (circles) or $B-\theta_{F}$ (crosses) JPDFs. Points to the right of the one-to-one line have a smaller error than the statistical method. 


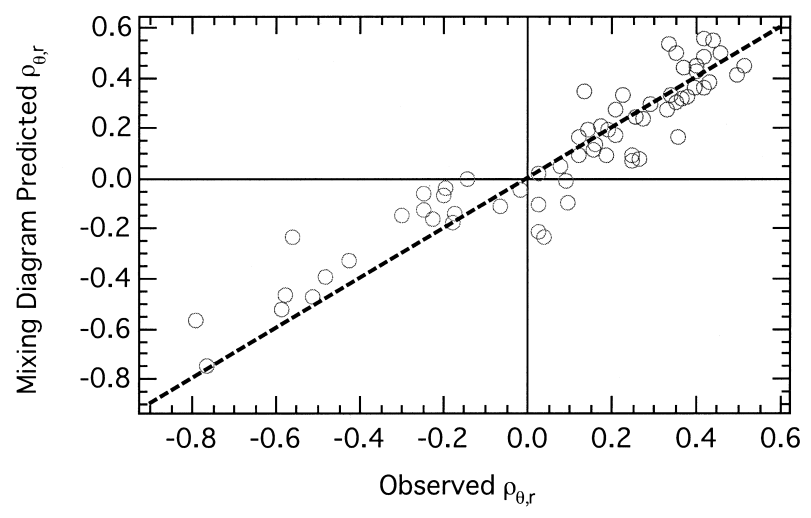

FIG. 13. Mixing diagram predicted $\rho_{\theta, r}$ vs observed $\rho_{\theta, r}$ for BLX96 flight legs. The solid line is the 1:1 line.

than the statistical method because the tilt of the JFD is a function of the slopes of the surface and entrainment-zone axes. Second, only $\sigma_{\text {ez }}$ and $\sigma_{\text {sfc }}$ must be parameterized in the mixing diagram method, compared to $\sigma_{\theta}, \sigma_{r}$, and $\rho_{\theta, r}$ that must be parameterized in the statistical method. But, if (18a) and (18b) are used to estimate $\sigma_{\text {ez }}$ and $\sigma_{\text {sfc }}$, then a third variable $\left(\rho_{\theta, r}\right)$ is needed and both the statistical method and the mixing diagram method depend on $\sigma_{\theta}, \sigma_{r}$, and $\rho_{\theta, r}$. At first glance, inclusion of $\rho_{\theta, r}$ would seem to reduce the advantage of the mixing diagram method over the statistical method. Closer examination of the terms in the equations for $\sigma_{\text {ez }}^{2}[(18 \mathrm{a})]$ and $\sigma_{\text {sfc }}^{2}[(18 b)]$ indicate that, for BLX96, the term that includes $\rho_{\theta, r}$ is nearly an order of magnitude smaller than the other terms, so the dependence of $\sigma_{\mathrm{ez}}^{2}$ and $\sigma_{\mathrm{sfc}}^{2}$ on $\rho_{\theta, r}$ is weak and the mixing diagram method is not sensitive to the observed value of $\rho_{\theta, r}$.

Given the weak dependence of $\sigma_{\mathrm{ez}}^{2}$ and $\sigma_{\mathrm{sfc}}^{2}$ on the observed value of $\rho_{\theta, r}$, the JPDFs found using the mixing diagram method can be used to predict $\rho_{\theta, r}$. There is good agreement between $\rho_{\theta, r}$ predicted from the mixing diagram method JPDF and $\rho_{\theta, r}$ observed during BLX96 (Fig. 13). This results shows that the axes selected by the mixing diagram method account for a significant amount of the observed correlation between $\theta$ and $r$. For cases where $\rho_{\theta, r}$ calculated from the BLX96 observations and $\rho_{\theta, r}$ predicted from the mixing diagram JPDFs are both negative, $\rho_{\theta, r}$ predicted from the mixing diagram JPDFs is slightly smaller than the observed. It is not clear why this is the case, but a number of these observations occur for legs near the top of the boundary layer. This could be related to errors in the entrainmentzone slope, which are interpolated to the time of the relevant flight leg. The skill of the mixing diagram predictions of $\rho_{\theta, r}$ is a significant advantage because $\rho_{\theta, r}$ may not be universal.

\section{Conclusions}

A new field experiment was conducted in which JFDs of $\theta$ versus $r$ were measured at heights ranging from
0.02 to $0.8 z_{i}$ in the daytime convective boundary layer. Results from BLX96 were consistent with findings of SSZ. It was found that JFDs near the surface had a positive tilt, in other words, warm parcels also tended to be relatively moist, while those JFDs measured higher in the mixed layer had a negative tilt because warm (entrained) parcels tended to be relatively dry. Each distribution was found to be skewed, and more peaked than a Gaussian distribution.

A new parameterization to describe the tilt of the JFDs was introduced. This new parameterization describes the mixed-layer JFDs as a mixture of air originating from near the surface, from the entrainment zone and from the environment. The relationship of these three source regions was used to tilt the JFDs. Similarity relationships were derived to describe the spread of the JFD along each axis. Using a calibration subset of observations from BLX96 the derived parameterizations were compared to a verification subset, also from BLX96. Fortunately, BLX96 flights were flown at a three different locations, so our findings are more likely applicable to other relatively flat geographic locations.

This new parameterization was compared to two classic parameterizations. The new parameterization was more successful than the method of SSZ, and about as successful as that of WM92. The primary benefit of the new parameterization are the physical relationships used to predict the tilt of the JFD and skill predicting $\rho_{\theta, r}$ based only on the slopes of the two new axes. The primary disadvantage is the requirement of the two additional variables: the slope of the surface and entrainment zone axes.

Acknowledgments. This work was completed as part of the lead authors doctoral research at the University of British Columbia (UBC), where funding was provided by a UBC Graduate Fellowship and the Canadian Climate Research Network through grants from the Natural Science and Engineering Research Council (NSERC), Meteorological Service of Canada (MSC), and the Canadian Foundation for Climate and Atmospheric Science. Additional support was provided by the Geophysical Disaster Computational Fluid Dynamics Centre, other grants from NSERC and Environment Canada, and a Laboratory Directed Research and Development Program of the U.S. Department of Energy under Contract DE-AC06-76RLO at the Pacific Northwest National Laboratory, which is operated for the U.S. Department of Energy by Battelle Memorial Institute. The BLX96 field program was funded by the U.S. National Science Foundation (NSF) under Grant ATM9411467. E. Santoso and J. Hacker are thanked for their work as airborne scientists during BLX96. The staff and flight crew of the University of Wyoming King Air aircraft (sponsored by NSF) helped to make BLX96 a success. The comments of three anonymous reviewers greatly improved this manuscript. 


\section{APPENDIX}

\section{Slopes Used in the $B-\theta_{F}$ Method}

Schrieber et al. (1996) derived equations for $\theta^{\prime}$ and $r^{\prime}$, the perturbation values from the means, as [their (4) and (5)]

$$
\begin{aligned}
\theta^{\prime} & =\frac{B \theta_{F}}{1+B}, \quad \text { and } \\
r^{\prime} & =\frac{\gamma \theta_{F}}{1+B},
\end{aligned}
$$

where $\gamma=C_{p} / L_{v}$ is the psychrometric constant and $\theta_{F}$ is the solar forcing (defined in section 5). They modified these equations to give slopes in terms of $\theta_{v}$ and $z_{\mathrm{LCL}}$. However, (A1) and (A2) can be combined to give the slopes in terms of $\theta$ and $r$. Like the work of Schrieber et al. (1996), lines of constant $B$ can be found by dividing (A2) by (A1):

$$
r^{\prime} / \theta^{\prime}=\gamma / B
$$

Lines of constant solar forcing can also be found from (A1) and (A2). First, (A2) is solved in terms of B. This value is substituted into (A1) leaving

$$
r^{\prime}=-\gamma \theta^{\prime}+\gamma \theta_{F},
$$

which is the equation for the $\theta_{F}$ lines. In $\theta$ versus $r$ space all lines of constant solar forcing have the same slope $-\gamma$. This result is different than lines of constant solar forcing in $\theta_{v}$ versus $z_{\mathrm{LCL}}$ space, which are a function of the reference state. Figure A1 shows an example of (A3) and (A4) plotted in $\theta$ versus $r$ space. Figure A1 appears to be a mirror image of Schrieber et al.'s Fig. 2 because large values of $z_{\mathrm{LCL}}$ correspond to small values of $r$.
REFERENCES

Ball, F. K., 1960: Control of inversion height by surface heating. Quart. J. Roy. Meteor. Soc., 86, 483-494.

Berg, L. K., 2002: A simple parameterization coupling the convective daytime boundary layer and fair-weather cumuli. Ph.D. dissertation. Atmospheric Science Programme, Department of Earth Sciences, The University of British Columbia, 166 pp. [Available from The University of British Columbia, 6339 Stores Rd., Vancouver, BC, V6T 1Z4, Canada.]

_ - R. B. Stull, E. Santoso, and J. P. Hacker, 1997: Boundary layer experiment-1996, airborne scientist flight log. Bound. Layer Tech. Rep. 97-1, 116 pp. [Available from Roland Stull, Atmospheric Science Programme, Department of Earth and Ocean Sciences, University of British Columbia, 6339 Stores Rd., Vancouver, BC V6T-1Z4, Canada.]

Betts, A. K., 1975: Parametric interpretation of trade-wind cumulus budget studies. J. Atmos. Sci., 32, 1934-1945.

—_ 1976: Modeling subcloud layer structure and interaction with a shallow cumulus layer. J. Atmos. Sci., 33, 2363-2382.

— 1982: Saturation point analysis of moist convective overturning. J. Atmos. Sci., 39, 1484-1505.

__ 1984: Boundary layer thermodynamics of a high plains severe storm. Mon. Wea. Rev., 112, 2199-2211.

_ 1985 : Mixing line analysis of clouds and cloudy boundary layers. J. Atmos. Sci., 42, 2751-2763.

, 1992: FIFE atmospheric boundary layer budget methods. $J$. Geophys. Res., 97, 18 523-18 531.

- , and B. A. Albrecht, 1987: Conserved variable analysis of the convective boundary layer thermodynamic structure over tropical oceans. J. Atmos. Sci., 44, 83-99.

Coulman, C. E., 1978: Boundary layer evolution and nocturnal inversion dispersal-Part II. Bound.-Layer Meteor., 14, 493-513.

Crum, T. D., and R. B. Stull, 1987: Field measurements of the amount of surface layer air versus height in the entrainment zone. $J$. Atmos. Sci., 44, 2743-2753.

Cuijpers, J. W. M., and A. A. M. Holtslag, 1998: Impact of skewness and nonlocal effects on scalar buoyancy fluxes in convective boundary layers. J. Atmos. Sci., 55, 151-162.

Deardorff, J. W., and G. E. Willis, 1985: Further results from a laboratory model of the convective planetary boundary layer. Bound.-Layer Meteor., 32, 205-236.

Greenhut, G. K., and S. J. S. Khalsa, 1982: Updraft and downdraft

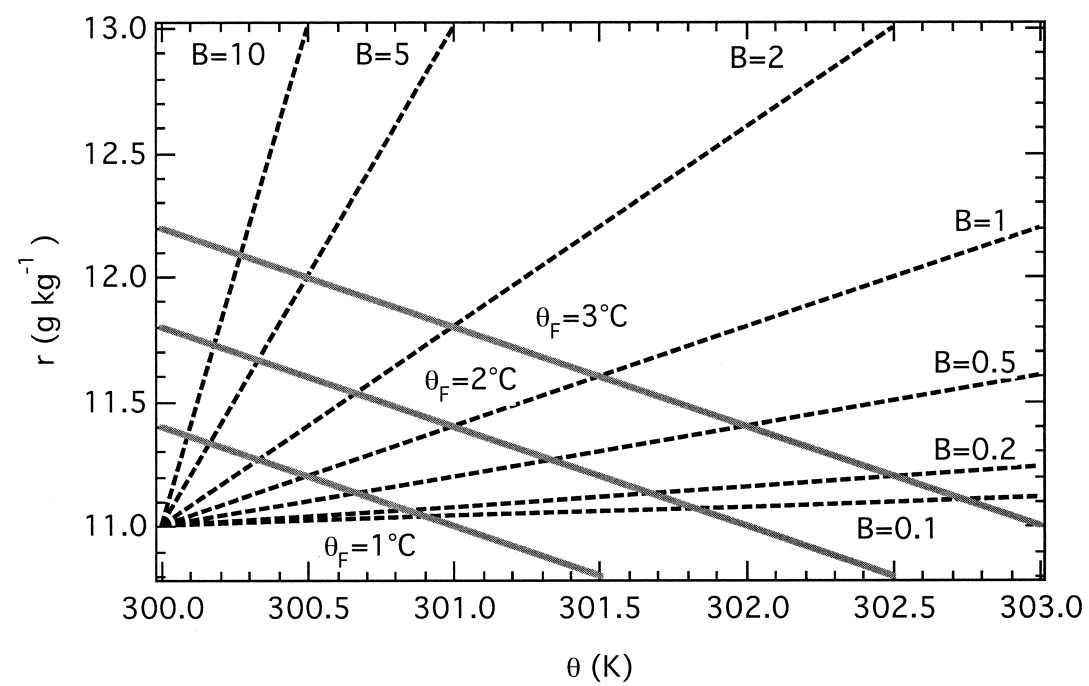

FIG. A1. Axes of $B$ (dashed lines) and $\theta_{F}$ (solid lines) for a range of values for a reference state defined as $\theta=300 \mathrm{~K}$ and $r=11 \mathrm{~g} \mathrm{~kg}^{-1}$, after Schrieber et al. (1996). 
events in the atmospheric boundary layer over the equatorial Pacific Ocean. J. Atmos. Sci., 39, 1803-1818.

- , and — 1987: Convective elements in the marine atmospheric boundary layer. Part I: Conditional sampling statistics. J. Climate Appl. Meteor., 26, 813-822.

Grossman, R. L., 1984: Bivariate conditional sampling of moisture flux over a tropical ocean. J. Atmos. Sci., 41, 3238-3252.

Hanson, H. P., 1984: On mixed layer modeling of the stratocumulustopped marine boundary layer. J. Atmos. Sci., 41, 1226-1234.

Holland, J. Z., 1973: A statistical method for analyzing wave shapes and phase relationships of fluctuationg geophysical variables. $J$. Phys. Oceanogr., 3, 139-155.

Khalsa, S. J. S., and G. K. Greenhut, 1985: Conditional sampling of updrafts and downdrafts in the marine atmospheric boundary layer. J. Atmos. Sci., 42, 2550-2562.

Larson, V. E., R. Wood, P. R. Field, J.-C. Golaz, T. H. Vonder Haar, and W. R. Cotton, 2001: Small-scale and mesoscale variability of scalars in cloudy boundary layers: One-dimensional probability density functions. J. Atmos. Sci., 58, 1978-1994.

Lenschow, D. H., 1970: Airplane measurements of the planetary boundary layer structure. J. Appl. Meteor., 9, 874-884.

- , and P. L. Stephens, 1980: The role of thermals in the convective boundary layer. Bound.-Layer Meteor., 19, 509-532.

Lewellen, W. S., and S. Yoh, 1993: Binormal model ensemble partial cloudiness. J. Atmos. Sci., 50, 1228-1237.

Mahrt, L., and J. Paumier, 1982: Cloud-top entrainment instability observed in AMTEX. J. Atmos. Sci., 39, 622-634.

- , and -1984 : Heat transport in the atmospheric boundary layer. J. Atmos. Sci., 41, 3061-3075.

Manton, J. R., 1975: Penetrative convection due to a field of thermals. J. Atmos. Sci., 32, 2272-2277.

Nicholls, S., and M. A. LeMone, 1980: The fair weather boundary layer in GATE: The relationship of subcloud fluxes and structure to the distribution and enhancement of cumulus clouds. J. Atmos. Sci., 37, 2051-2067.

Paluch, I. R., 1979: Entrainment mechanism in Colorado cumuli. J. Atmos. Sci., 36, 2467-2478.

Press, W. H., S. A. Teukolsky, W. T. Vetterling, and B. P. Flannery, 1992: Numerical Recipes in C: The Art of Scientific Computing. Cambridge University Press, 994 pp.

Randall, D. A., Q. Shao, and C.-H. Moeng, 1992: A second-order bulk boundary-layer model. J. Atmos. Sci., 49, 1903-1923.

Santoso, E., and R. B. Stull, 1998: Wind and temperature profiles in the radix layer, the bottom fifth of the convective boundary layer. J. Appl. Meteor., 37, 545-558.

, and — 2001: Similarity equations for wind and temperature profiles in the radix layer, at the bottom of the convective boundary layer. J. Atmos. Sci., 58, 1446-1464.

Schrieber, K., R. Stull, and Q. Zhang, 1996: Distributions of surfacelayer buoyancy versus lifting condensation level over a heterogeneous land surface. J. Atmos. Sci., 53, 1086-1107.

Schumann, U., and C.-H. Moeng, 1991: Plume fluxes in the clear and cloudy convective boundary layers. J. Atmos. Sci., 48, 17461757 .

Siebesma, A. P., and J. W. M. Cuijpers, 1995: Evaluation of parametric assumptions for shallow cumulus convection. J. Atmos. Sci., $\mathbf{5 2}$, 650-666.

Sorbjan, Z., 1991: Evaluation of local similarity functions in the convective boundary layer. J. Appl. Meteor., 30, 1565-1583.

Stokes, G. M., and S. E. Swartz, 1994: The atmospheric radiation measurement (ARM) program: Programmatic background and design of the cloud and radiation testbed. Bull. Amer. Meteor. Soc., 75, 1201-1221.

Stull, R. B., 1994: A convective transport theory for surface fluxes. J. Atmos. Sci., 51, 3-22.

— Experiment 1996 (BLX96). Bull. Amer. Meteor. Soc., 78, 11491158.

Wang, S., and B. Stevens, 2000: Top-hat representation of turbulence statistics in cloud-topped boundary layers: A large eddy simulation study. J. Atmos. Sci., 57, 423-441.

Williams, A. G., and J. M. Hacker, 1992: The composite shape and structure of coherent eddies in the convective mixed layer. Bound.-Layer Meteor., 61, 213-245.

Willis, G. E., and J. W. Deardorff, 1974: A laboratory model of the unstable planetary boundary layer. J. Atmos. Sci., 31, $1297-$ 1307.

Wyngaard, J. C., and M. A. LeMone, 1980: Behavior of the refractive index structure parameter in the entraining convective boundary layer. J. Atmos. Sci., 37, 1573-1585.

— , and C.-H. Moeng, 1992: Parameterizing turbulent diffusion through the joint probability density. Bound.-Layer Meteor., 60, $1-13$.

— W. T. Pennell, D. H. Lenschow, and M. A. LeMone, 1978: The temperature-humidity covariance budget in the convective boundary layer. J. Atmos. Sci., 35, 47-58.

Xu, K.-M., and D. A. Randall, 1996: Evaluation of statistically based cloudiness parameterizations used in climate models. J. Atmos. Sci., 53, 3103-3119.

Young, G. S., 1988: Turbulence structure of the convective boundary layer. Part II: Phoenix 78 aircraft observations of thermals and their environment. J. Atmos. Sci., 45, 727-735. 